\title{
Cyclotomic polynomials with large coefficients
}

\author{
by \\ Helmut Maier (Athens, Ga.) \\ Dedicated to Paul Erdös \\ on the occasion of his eightieth birthday
}

1. Introduction. Let $\Phi_{n}(z)=\sum_{m=0}^{\varphi(n)} a(m, n) z^{m}$ be the $n$th cyclotomic polynomial. Let

$$
A(n)=\max _{0 \leq m \leq \varphi(n)}|a(m, n)| \quad \text { and } \quad S(n)=\sum_{0 \leq m \leq \varphi(n)}|a(m, n)| .
$$

The coefficients $a(m, n)$ and especially $A(n)$ and $S(n)$ have been the subject of numerous investigations (see [1] and the references given there). Until recently all these investigations concerned very thin sets of integers $n$. In [3] the author could establish a property valid for a set of integers of asymptotic density 1 . Let $\varepsilon(n)$ be any function defined for all positive integers such that $\lim _{n \rightarrow \infty} \varepsilon(n)=0$. Then $S(n) \geq n^{1+\varepsilon(n)}$ for a set of integers of asymptotic density 1 . Here we deal with properties valid for sequences of positive lower density.

Theorem. For any $N>0$, there are $c(N)>0$ and $x_{0}(N) \geq 1$ such that

$$
\operatorname{card}\left\{n \leq x: A(n) \geq n^{N}\right\} \geq c(N) x,
$$

for all $x \geq x_{0}(N)$.

2. A certain set of candidates. Let $N>0$ be given. In this section we identify a certain set of integers in which a large subset will later be shown to have $A(n) \geq n^{N}$. To describe the set, we fix a positive odd integer

$$
K=K(N)
$$

(to be determined later) and set

$$
L=20 K, \quad \delta=\frac{1}{100 L}, \quad \varepsilon=\frac{\delta}{L^{2}} .
$$

The author was supported in part by an NSF grant. 
The letter $p$ always denotes prime numbers and $\omega(m)$ denotes the number of distinct prime factors of $m$. Basic for our construction is the set $S$.

Let

$$
\begin{aligned}
S=S(N, x)=\left\{n=m p_{1} \ldots p_{L}\right. & \leq x: x^{\frac{1-\delta}{L}-\varepsilon}<p_{i} \leq x^{\frac{1-\delta}{L}+\varepsilon} \\
\mu(m)=\mu(n) & =1, \omega(m) \leq(1+\varepsilon)(\log \log x)\} .
\end{aligned}
$$

Lemma. For each $N$, there are effectively computable constants $c_{0}(N)$ $>0$ and $x_{0}(N)>0$ such that for all $x \geq x_{0}(N)$,

$$
\operatorname{card} S \geq c_{0}(N) x \text {. }
$$

Pr o o f. Let $\mathcal{T}=\mathcal{T}(x)$ be the set of $n \leq x$ which have all of the properties of elements of $S$, but the condition $\mu(n)=1$ fails. This implies that at least two of the primes $p_{1}, \ldots, p_{L}$ are the same. We have

$$
\operatorname{card} S \geq \frac{1}{L !} \sum_{p_{1}}^{\prime} \cdots \sum_{p_{L}}^{\prime} \sum_{\substack{m \leq x / p_{1} \ldots p_{L} \\ \mu(m)=1, \omega(m) \leq(1+\varepsilon) \log \log x}} 1-\operatorname{card} \mathcal{T}
$$

where $\sum^{\prime}$ denotes a sum over $p_{i}$ with $x^{\frac{1-\delta}{L}-\varepsilon}<p_{i} \leq x^{\frac{1-\delta}{L}+\varepsilon}$.

Clearly

$$
\operatorname{card} \mathcal{T} \leq x \sum_{p}^{\prime} \frac{1}{p^{2}}=o(x) \quad \text { for } x \rightarrow \infty .
$$

From [2] and [4] we know that the inner sum in (2.3) is at least $x / 10 p_{1} \ldots p_{L}$ for all $x \geq x_{1}(N)$, where $x_{1}(N)$ is a constant depending only on the choice of $N$. Thus

$$
\operatorname{card} S \geq \frac{x}{10 L !}\left(\sum^{\prime} \frac{1}{p}\right)^{L}-o(x) \quad \text { for } x \rightarrow \infty .
$$

Now

$$
\sum^{\prime} \frac{1}{p}=\log \left(\frac{1-\delta}{L}+\varepsilon\right)-\log \left(\frac{1-\delta}{L}-\varepsilon\right)+O\left(\frac{1}{\log x}\right),
$$

so there is some number $c_{1}(N)$ such that

$$
\sum^{\prime} \frac{1}{p} \geq c_{1}(N)>0
$$

for all $x \geq x_{2}(N)$, where $x_{2}(N)$ is a constant depending only on $N$. The lemma now follows from (2.4).

3. Investigation of $\log \left|\Phi_{n}(z)\right|$ on the unit circle. We start with the well-known identity

$$
\Phi_{n}(z)=\prod_{d \mid n}\left(1-z^{d}\right)^{\mu(n / d)}
$$


for all complex $z$ for which both sides are defined. We write $e^{2 \pi i \alpha}=e(\alpha)$ and obtain

$$
\log \left|\Phi_{n}(e(\alpha))\right|=\sum_{d \mid n} \mu\left(\frac{n}{d}\right) \log |1-e(\alpha d)| .
$$

To show that $A(n)$ is large it would suffice to show there is some $\alpha$ with $\log \left|\Phi_{n}(e(\alpha))\right|$ large. The terms on the right of (3.2) will be large for $\mu(n / d)$ $=-1$ and $\|\alpha d\|$ small. (Here $\|\cdot\|$ denotes the distance to the nearest integer.) Simple choices for the pair $(\alpha, d)$ however do not work because of a certain cancellation effect which has already been described in [3]. We repeat its description for the convenience of the reader.

Assume $r \mid n / d, \mu(n / d)=-1, \mu(n) \neq 0, \omega(r) \geq 2$ and $\alpha d=k+\varrho$ with $k$ an integer, $|\varrho| \leq 1 / 2$. Thus $|\varrho|=\|\alpha d\|$. Also assume that $|r \varrho| \leq$ $1 / 2$. For $t \mid r$ we have $e(\alpha d t)=e(t \varrho)=1+2 \pi i \varrho t+O\left((\varrho t)^{2}\right)$. This implies $\log |1-e(\alpha d t)|=\log (\varrho t)+O(1)$. Thus

$$
\begin{aligned}
\sum_{t \mid r} \mu\left(\frac{n}{d t}\right) \log \mid 1 & -e(\alpha d t) \mid \\
= & \sum_{t \mid r} \mu\left(\frac{n}{d t}\right)(\log \varrho+\log t)+O\left(\sum_{t \mid r} 1\right)=O\left(\sum_{t \mid r} 1\right),
\end{aligned}
$$

where we use

$$
\sum_{t \mid r} \mu(t)=0 \quad \text { and }(\text { for } \omega(r) \geq 2) \quad \sum_{t \mid r} \mu(t) \log t=0 .
$$

Thus the large contribution $\mu(n / d) \log |1-e(\alpha d)|$ is cancelled by other terms.

A method to avoid this cancellation effect is to choose $\alpha$ and $d$ such that for $t<t_{0}$ we have $|\varrho t| \leq 1 / 2$ but for $t \geq t_{0}$, $|\varrho t|>1 / 2$. This leads to estimates of incomplete convolutions

$$
\sum_{\substack{t \mid r \\ t<t_{0}}} \mu\left(\frac{n}{d t}\right)(\log \varrho+\log t)
$$

which can be made large by an appropriate choice of $d, r, t_{0}$ and $\alpha$. For the remaining sum

$$
\sum_{\substack{t \mid r \\ t \geq t_{0}}} \mu\left(\frac{n}{d t}\right) \log |1-e(\alpha d t)|
$$

we have to show that the terms are small for appropriate choice of $\alpha$. This will be done by showing that $\|\alpha d t\|$ is not too small. 
Definitions. Let $S\left(m_{0}\right)=\left\{n \in S: n=m_{0} p_{1} \ldots p_{L}\right\}, y_{0}=x^{-\frac{1}{L}(K+1-\delta)}$. For each $m_{0}$ we define an interval

$$
I\left(m_{0}\right)=\left[m_{0}^{-1}+m_{0}^{-1} y_{0}, m_{0}^{-1}+2 m_{0}^{-1} y_{0}\right] .
$$

For $n \in S$ with $n=m p_{1} \ldots p_{L}$, set $\Pi(n)=p_{1} p_{2} \ldots p_{L}$. If $n \in S\left(m_{0}\right)$, we write

$$
\log \left|\Phi_{n}(e(\alpha))\right|=\sum_{0}+\sum_{1}+\sum_{2}
$$

where

$$
\begin{aligned}
& \sum_{0}=\sum_{\substack{d=m_{0} t, t \mid \Pi(n) \\
\omega(t) \leq K}} \mu\left(\frac{n}{d}\right) \log |1-e(\alpha d)|, \\
& \sum_{1}=\sum_{\substack{m^{*} \mid m_{0} \\
m^{*} \neq m_{0}}} \mu\left(\frac{n}{m^{*}}\right) \log \left|1-e\left(\alpha m^{*}\right)\right|, \\
& \sum_{2}=\sum_{\substack{\text { all other divisors } \\
d \mid n}} \mu\left(\frac{n}{d}\right) \log |1-e(\alpha d)| .
\end{aligned}
$$

We shall investigate these three sums for $\alpha \in I\left(m_{0}\right)$.

4. The main part $\sum_{0}$. Let $t \mid \Pi(n)$ with $\omega(t)=K-l, 0 \leq l \leq K$. Then $t$ is the product of $K-l$ distinct primes from $\left[x^{\frac{1-\delta}{L}-\varepsilon}, x^{\frac{1-\delta}{L}+\varepsilon}\right]$. Therefore

$$
t \in\left[x^{\frac{K-l}{L}(1-\delta)-(K-l) \varepsilon}, x^{\frac{K-l}{L}(1-\delta)+(K-l) \varepsilon}\right] .
$$

Moreover, if $\alpha \in I\left(m_{0}\right)$, then $\alpha m_{0} t \in\left[t+t y_{0}, t+2 t y_{0}\right]$, so that $\left\{\alpha m_{0} t\right\} \in$ $\left[t y_{0}, 2 t y_{0}\right]$, where $\{\cdot\}$ means fractional part. We write $\left\{\alpha m_{0} t\right\}=\eta t y_{0}$ with $1 \leq \eta \leq 2$. We have $e\left(\alpha m_{0} t\right)=e\left(\left\{\alpha m_{0} t\right\}\right)=1+2 \pi i \eta t y_{0}+O\left(\left(t y_{0}\right)^{2}\right)$ and thus

$$
\log \left|1-e\left(\alpha m_{0} t\right)\right|=\log t y_{0}+O(1) .
$$

From (4.1) and the definition of $y_{0}$ we get

$$
t y_{0} \in\left[x^{-\frac{l+1}{L}-(K-l-1) \frac{\delta}{L}-(K-l) \varepsilon}, x^{-\frac{l+1}{L}-(K-l-1) \frac{\delta}{L}+(K-l) \varepsilon}\right] .
$$

For $l=0$, that is, for $\omega(t)=K$, we use the upper bound in (4.3) and together with (4.2) we get

$$
\log \left|1-e\left(\alpha m_{0} t\right)\right| \leq\left(-\frac{1}{L}-(K-1) \frac{\delta}{L}+K \varepsilon\right) \log x+O(1) \leq-\frac{1}{L} \log x,
$$

for $x$ sufficiently large. There are $\left(\begin{array}{l}L \\ K\end{array}\right)$ divisors $t \mid \Pi(n)$ with $\omega(t)=K$ and 
for each we have $\mu\left(n /\left(m_{0} t\right)\right)=-1$. Thus we get

$$
\sum_{\substack{t \mid \Pi(n) \\
\omega(t)=K}} \mu\left(\frac{n}{m_{0} t}\right) \log \left|1-e\left(\alpha m_{0} t\right)\right| \geq\left(\begin{array}{c}
L \\
K
\end{array}\right) L^{-1} \log x .
$$

For $1 \leq l \leq K$, that is, $\omega(t)=K-l$, from (4.2) and (4.3) we get

$|\log | 1-e\left(\alpha m_{0} t\right)|| \leq\left(\frac{l+1}{L}+(K-l-1) \frac{\delta}{L}+(K-l) \varepsilon\right) \log x+O(1) \leq \frac{3 l}{L} \log x$,

for $x$ sufficiently large. Since there are $\left(\begin{array}{c}L \\ K-l\end{array}\right)$ divisors $t \mid \Pi(n)$ with $\omega(t)=$ $K-l$ we get

$$
\left|\sum_{\substack{t \mid \Pi(n) \\
\omega(t)=K-l}} \mu\left(\frac{n}{m_{0} t}\right) \log \right| 1-e\left(\alpha m_{0} t\right) \mid \leq \frac{3 l}{L}\left(\begin{array}{c}
L \\
K-l
\end{array}\right) \log x .
$$

We study the ratio of these upper bounds for consecutive $l$-values. For $l \geq 1$,

$$
\begin{aligned}
\frac{3(l+1)}{L}\left(\begin{array}{c}
L \\
K-l-1
\end{array}\right) / \frac{3 l}{L}\left(\begin{array}{c}
L \\
K-l
\end{array}\right) & =\left(1+\frac{1}{l}\right) \frac{K-l}{L-(K-l)+1} \\
& <\frac{2 K}{L-K}=\frac{2}{19} .
\end{aligned}
$$

From this, (4.4) and (4.5) we obtain for $x$ sufficiently large

$$
\begin{aligned}
\sum_{0} & \geq \frac{1}{L}\left(\begin{array}{c}
L \\
K
\end{array}\right) \log x-\frac{3}{L}\left(\begin{array}{c}
L \\
K-1
\end{array}\right) \log x \sum_{i=0}^{\infty}\left(\frac{2}{19}\right)^{i} \\
& =\frac{1}{L}\left(\begin{array}{c}
L \\
K
\end{array}\right) \log x-\frac{57}{17} \cdot \frac{1}{L}\left(\begin{array}{c}
L \\
K-1
\end{array}\right) \log x \\
& =\left(1-\frac{57}{17} \frac{K}{L-K+1}\right) \frac{1}{L}\left(\begin{array}{c}
L \\
K
\end{array}\right) \log x>\frac{14}{17 L}\left(\begin{array}{c}
L \\
K
\end{array}\right) \log x
\end{aligned}
$$

5. The divisors of $m_{0}$. Our aim now is to show that $\sum_{1}$ is small for $n \in S, \alpha \in I\left(m_{0}\right)$. By definition we have

$$
\sum_{1}=\sum_{\substack{m^{*} \mid m_{0} \\ m^{*} \neq m_{0}}} \mu\left(\frac{n}{m^{*}}\right) \log \left|1-e\left(\alpha m^{*}\right)\right| .
$$

Note that for $\alpha \in I\left(m_{0}\right)$ and $m^{*} \mid m_{0}, m^{*}<m_{0}$ we have $0<\alpha m^{*}<1$. Thus

$$
e\left(\alpha m^{*}\right)=1+2 \pi i \alpha m^{*}+O\left(\left(\alpha m^{*}\right)^{2}\right) .
$$

From this we get

$$
\left|1-e\left(\alpha m^{*}\right)\right|=2 \pi \alpha m^{*}\left(1+O\left(\alpha m^{*}\right)\right)
$$


and so

$$
\log \left|1-e\left(\alpha m^{*}\right)\right|=\log m^{*}+\log \alpha+O(1) .
$$

Thus for all $n \in S$,

$$
\sum_{1}=\mu\left(\frac{n}{m_{0}}\right) \sum_{\substack{m^{*} \mid m_{0} \\ m^{*} \neq m_{0}}} \mu\left(\frac{m_{0}}{m^{*}}\right)\left(\log m^{*}+\log \alpha\right)+O\left((\log x)^{(1+\varepsilon) \log 2}\right),
$$

since $\omega\left(m_{0}\right) \leq(1+\varepsilon) \log \log x$.

We have (since $\mu\left(m_{0}\right)=1$ implies $m_{0}$ is not a prime or prime power)

$$
\sum_{\substack{m^{*} \mid m_{0} \\ m^{*} \neq m_{0}}} \mu\left(\frac{m_{0}}{m^{*}}\right) \log m^{*}=-\log m_{0}
$$

and

$$
\sum_{\substack{m^{*} \mid m_{0} \\ m^{*} \neq m_{0}}} \mu\left(\frac{m_{0}}{m^{*}}\right) \log \alpha=-\log \alpha .
$$

Since $\log \left(\alpha m_{0}\right) \ll 1$, this yields

$$
\sum_{1} \ll(\log x)^{(1+\varepsilon) \log 2} \quad \text { for } n \in S, \alpha \in I\left(m_{0}\right) .
$$

6. The divisors $d=m^{*} t$ with $\omega(t) \leq K$. The remaining divisors in $\sum_{2}$ are of two kinds. The first kind are of the form $m^{*} t$ with $m^{*} \mid m_{0}$, $m^{*}<m_{0}, t \mid \Pi(n)$ and $1 \leq \omega(t) \leq K$. We treat the contribution of these divisors in this section, leaving the treatment for the remaining divisors, which are of the form $m^{*} t$ with $\omega(t)>K$, for the final section.

Let $C>0$ be a constant that we will soon choose as a large absolute constant. If we have $\left\|m_{0}^{-1} m^{*} t\right\|>2(\log x)^{-C}$ for $\omega(t) \leq K$, then we also have

$$
\left\|\alpha m^{*} t\right\| \geq(\log x)^{-C}
$$

for all $\alpha \in I\left(m_{0}\right)$. Indeed,

$$
\left|\alpha m^{*} t-m_{0}^{-1} m^{*} t\right| \leq 2 m_{0}^{-1} y_{0} m^{*} t \leq y_{0} t=o\left((\log x)^{-C}\right)
$$

for any $C$.

We study the exceptional set

$$
\begin{aligned}
S_{E}\left(m_{0}\right)= & \left\{n \in S\left(m_{0}\right):\left\|m_{0}^{-1} m^{*} t\right\| \leq 2(\log x)^{-C}\right. \\
& \left.\quad \text { for some } m^{*}\left|m_{0}, m^{*}<m_{0}, t\right| \Pi(n), 1 \leq \omega(t) \leq K\right\} .
\end{aligned}
$$

We shall replace the inequality with a congruence. Let $n \in S_{E}$ and suppose $\left\|m_{0}^{-1} m^{*} t\right\| \leq 2(\log x)^{-C}$. Let $m_{0}^{-1} m^{*} t=k+\varrho$ where $k$ is an integer and 
$|\varrho| \leq 2(\log x)^{-C}$. Then

$$
t=\frac{m_{0}}{m^{*}} k+\frac{m_{0}}{m^{*}} \varrho .
$$

Note that $r:=\left(m_{0} / m^{*}\right) \varrho$ is an integer. Thus

$$
t \equiv r \bmod \frac{m_{0}}{m^{*}}, \quad|r| \leq 2 \frac{m_{0}}{m^{*}}(\log x)^{-C} .
$$

We estimate the cardinality of $S_{E}\left(m_{0}\right)$ by writing $S_{E}\left(m_{0}\right)$ as a union of subsets. For a given $m^{*} \mid m_{0}$ with $m^{*}<m_{0}$ and a given integer $g$ with $1 \leq g \leq K$, let

$$
\begin{aligned}
S_{E}\left(m_{0}, m^{*}, g\right)=\left\{n \in S\left(m_{0}\right): \|\right. & m_{0}^{-1} m^{*} t \| \leq 2(\log x)^{-C} \\
& \text { for some } t \mid \Pi(n) \text { with } \omega(t)=g\} .
\end{aligned}
$$

Note that if $n=m_{0} t u \in S_{E}\left(m_{0}, m^{*}, g\right)$ then $u \leq x /\left(m_{0} t\right)$ and

$$
x^{g\left(\frac{1-\delta}{L}-\varepsilon\right)}<t \leq x^{g\left(\frac{1-\delta}{L}+\varepsilon\right)}
$$

and (6.3) holds for some integer $r$. Thus

$$
\left|S_{E}\left(m_{0}, m^{*}, g\right)\right| \leq \sum_{t}^{*} \sum_{u \leq x /\left(m_{0} t\right)} 1 \leq \frac{x}{m_{0}} \sum_{t}^{* 1} \frac{1}{t}
$$

where $\sum^{*}$ denotes a sum over $t$ satisfying (6.4) and satisfying (6.3) for some integer $r$. Since $t \mid \Pi(n)$ and $\omega(t) \geq 1$ one has $t>m_{0} / m^{*}$, so that possible solutions of (6.3) with $t \leq m_{0} / m^{*}$ do not occur in the sum $\sum^{*}$. Thus for a fixed $r$, we have

$$
\sum_{t}^{*(r)} \frac{1}{t} \ll \frac{g \varepsilon \log x}{m_{0} / m^{*}}
$$

uniformly in $r$. Since each prime divisor of $t$ exceeds $m_{0} / m^{*}$, we see that $r=0$ is not a possibility in (6.3) and so the set of possible values of $r$ is empty when $2\left(m_{0} / m^{*}\right)(\log x)^{-C}<1$. Therefore

$$
\left|S_{E}\left(m_{0}, m^{*}, g\right)\right| \ll g \varepsilon \frac{m^{*}}{m_{0}^{2}} x \log x \sum_{|r| \leq 2\left|m_{0} / m^{*}\right|(\log x)^{-C}} 1 .
$$

Thus

$$
\begin{aligned}
\sum_{m_{0}}\left|S_{E}\left(m_{0}\right)\right| & \leq \sum_{m_{0}} \sum_{m^{*} \mid m_{0}} \sum_{g=1}^{K}\left|S_{E}\left(m_{0}, m^{*}, g\right)\right| \\
& \ll \sum_{m_{0}} \sum_{m^{*} \mid m_{0}} \frac{K^{2} \varepsilon}{m_{0}} x(\log x)^{1-C} \\
& <\sum_{m_{0}} \frac{\tau\left(m_{0}\right)}{m_{0}} x(\log x)^{1-C} \ll x(\log x)^{3-C} .
\end{aligned}
$$


7. The larger divisors. Here we study the divisors $d=m^{*} t$ with $m^{*}\left|m_{0}, t\right| \Pi(n), \omega(t)>K$. In contrast to the last section we here have to remove exceptional $\alpha$-values from $I\left(m_{0}\right)$.

Given a fixed triplet $\left(m_{0}, m^{*}, t\right)$ with $m^{*}\left|m_{0}, t\right| \Pi(n), \omega(t)>K$, we want an estimate for $\lambda E\left(m_{0}, m^{*}, t\right)$, where $E\left(m_{0}, m^{*}, t\right)=\left\{\alpha \in I\left(m_{0}\right)\right.$ : $\left.\left\|m^{*} t \alpha\right\| \leq(\log x)^{-C}\right\}$, and $\lambda$ denotes the Lebesgue measure. We have

$$
\begin{aligned}
& \lambda E\left(m_{0}, m^{*}, t\right)=\lambda\left\{\alpha \in I\left(m_{0}\right):\right. \\
& \left.\quad \alpha \in\left[\frac{k}{m^{*} t}-\frac{(\log x)^{-C}}{m^{*} t}, \frac{k}{m^{*} t}+\frac{(\log x)^{-C}}{m^{*} t}\right] \text { for some } k \in \mathbb{Z}\right\} .
\end{aligned}
$$

We determine the number of integers $k$ for which

$$
\left[\frac{k}{m^{*} t}-\frac{(\log x)^{-C}}{m^{*} t}, \frac{k}{m^{*} t}+\frac{(\log x)^{-C}}{m^{*} t}\right] \cap I\left(m_{0}\right) \neq \emptyset .
$$

From the definition of $I\left(m_{0}\right)$, such $k$-values satisfy

$$
\frac{m^{*} t}{m_{0}}+\frac{y_{0} m^{*} t}{m_{0}}-(\log x)^{-C} \leq k \leq \frac{m^{*} t}{m_{0}}+\frac{2 y_{0} m^{*} t}{m_{0}}+(\log x)^{-C} .
$$

Since $\omega(t)>K$, we have $y_{0} m^{*} t / m_{0} \rightarrow \infty$ as $x \rightarrow \infty$. Thus for large $x$, the number of integers $k$ satisfying (7.1) is at most $2 y_{0} m^{*} t / m_{0}$. Thus for large $x$, we have

$$
\lambda E\left(m_{0}, m^{*}, t\right) \leq \frac{2 y_{0}}{m_{0}(\log x)^{C}} .
$$

For $n \in S$, let

$$
J(n)=I\left(m_{0}\right)-\bigcup_{\substack{m^{*}\left|m_{0} \\ t\right| \Pi(n), \omega(t)>K}} E\left(m_{0}, m^{*}, t\right) .
$$

Thus from (7.2) we have

$$
\begin{aligned}
\lambda J(n) & \geq \frac{y_{0}}{m_{0}}-\sum_{\substack{m^{*}\left|m_{0} \\
t\right| \Pi(n), \omega(t)>K}} \frac{2 y_{0}}{m_{0}(\log x)^{C}} \\
& \geq \frac{y_{0}}{m_{0}}\left(1-\frac{2 \tau\left(m_{0}\right) 2^{L}}{(\log x)^{C}}\right) \geq \frac{y_{0}}{m_{0}}\left(1-2^{L+1}(\log x)^{1-C}\right)
\end{aligned}
$$

from the definition of $S$. Thus for $x$ large, we have $J(n) \neq \emptyset$.

We now use the results of this section and the previous section to estimate $\sum_{2}$. Let $n \in S-S_{E}$ and let $\alpha \in J(n)$. Then from (6.1) and the definition of $J(n)$, we have for each divisor $d$ of $n$ in the sum $\sum_{2}$ that

$$
\|\alpha d\| \geq(\log x)^{-C} \text {. }
$$


Thus for these values of $d$ and $\alpha$ we have

$$
|\log | 1-e(\alpha d)|| \ll \log \log x
$$

Recalling the definition of $\sum_{2}$ in (3.3) we conclude that

$$
\sum_{2} \ll \tau\left(m_{0}\right) 2^{L} \log \log x \ll(\log x)^{(1+2 \varepsilon) \log 2} .
$$

Combining this estimate with our estimates (4.6) and (5.1) for $\sum_{0}$ and $\sum_{1}$, we have for $x$ sufficiently large, $n \in S-S_{E}$, and $\alpha \in J(n)$,

$$
\log (n A(n)) \geq \log S(n) \geq \log \left|\Phi_{n}(e(\alpha))\right|>\frac{1}{2}\left(\begin{array}{c}
L \\
K
\end{array}\right) L^{-1} \log x .
$$

From Sections 2 and 6 we have $\left|S-S_{E}\right| \gg_{K} x$ for any fixed $K$. Thus by choosing $K$ sufficiently large, we have the Theorem.

Acknowledgments. The author wants to thank Professor Carl Pomerance for valuable advice concerning the presentation of this paper.

\section{References}

[1] P. T. Bateman, C. Pomerance and R. C. Vaughan, On the size of the coefficients of the cyclotomic polynomial, in: Topics in Classical Number Theory, Vol. I, G. Halász (ed.), Colloq. Math. Soc. János Bolyai 34, North-Holland, 1984, 171-202.

[2] G. H. Hardy and S. Ramanujan, The normal number of prime factors of a number n, Quart. J. Math. (Oxford) 48 (1917), 76-92.

[3] H. Maier, The coefficients of cyclotomic polynomials, in: Analytic Number Theory, Proc. Conf. in Honor of Paul T. Bateman, Progr. Math. 85, Birkhäuser, 1990, 349366.

[4] K. Prachar, Primzahlverteilung, Springer, Berlin 1957.

DEPARTMENT OF MATHEMATICS

UNIVERSITY OF GEORGIA

ATHENS, GEORGIA 30602

U.S.A. 